\title{
Generation of electromagnetic fields of extremely high intensity by coherent summation of Cherenkov superradiance pulses
}

\author{
N. S. Ginzburg ${ }^{1}$, A. W. Cross ${ }^{2}$, A. A. Golovanov ${ }^{1}$, G. A. Mesyats ${ }^{3}$, M. S. Pedos ${ }^{4}$, \\ A. D. R. Phelps ${ }^{2}$, I. V. Romanchenko 5 , V. V. Rostov $^{5}$, S. N. Rukin ${ }^{4}$, K. A. Sharypov ${ }^{4}$, \\ V. G. Shpak ${ }^{4}$, S. A. Shunailov ${ }^{4}$, M. R. Ulmaskulov ${ }^{4}$, M. I. Yalandin ${ }^{4}$, I. V. Zotova ${ }^{1}$ \\ ${ }^{1}$ Institute of Applied Physics, RAS, Nizhny Novgorod, Russia \\ ${ }^{2}$ Dept. of Physics, University of Strathclyde, Glasgow, UK \\ ${ }^{3}$ P. N. Lebedev Physical Institute, RAS, Moscow, Russia \\ ${ }^{4}$ Institute of Electrophysics, UB RAS, Ekaterinburg, Russia \\ ${ }^{5}$ Institute of High-Current Electronics, SB RAS, Tomsk, Russia \\ E-mail: zotova@appl.sci-nnov.ru
}

\begin{abstract}
We demonstrate both theoretically and experimentally the possibility of correlating the phase of a Cherenkov superradiance (SR) pulse to the sharp edge of a current pulse, when spontaneous emission of the electron bunch edge serves as the seed for SR processes. By division of the driving voltage pulse across several parallel channels equipped with independent cathodes we can synchronize several SR sources to arrange a two-dimensional array. In experiments carried out, coherent summation of radiation from four independent $8-\mathrm{mm}$ wavelength band SR generators with peak power $600 \mathrm{MW}$ resulted in the interference maximum of the directional diagram with an intensity that is equivalent to radiation from a single source with power $10 \mathrm{GW}$.
\end{abstract}

DOI: $10.1103 /$ PhysRevLett.115.114802

PACS numbers: 41.60.Bq. 84.40.Fe.

Numerous scientific and technological applications stimulate interest in the generation of ultra-high power coherent radiation. Approaches that can be suggested to achieve this goal include the generation of radiation by a single source with an oversized electrodynamic system. In this case special methods (for example, 2D distributed feedback [1,2]) are required to produce spatially coherent radiation. Another method is the synchronization of a large number of moderate-power sources using a master oscillator [3-5].

At the same time for short-pulse sources, in particular, for sources based on Cherenkov superradiance (SR) of extended electron bunches moving in a slow wave structure (SWS) [6,7], there is an alternative opportunity, associated with the correlating the phase of a radiated pulse to the sharp edge of a current pulse. In fact, spontaneous emission of the bunch edge serves as the seed for SR processes. It gives rise to the stimulated emission including electron self-bunching and subsequent radiation of the short high-power electromagnetic pulse. If identical current pulses are sent simultaneously to several channels, identical SR pulses will be generated and the coherent summation of their amplitudes is possible. For two channel radiation sources such a possibility has been experimentally demonstrated in Ref. [8]. However the physical model describing the transformation of spontaneous Cherenkov radiation (i.e. the radiation from the unperturbed moving particles without the reverse effect of the field [9]) to stimulated radiation is still missing. The 
development of such a model and the reporting of significant new experimental results is the main aim of this Letter. In experiments carried out, for the first time a two-dimensional array consisting of four synchronized Cherenkov SR generators has been realized, which allows extremely high electromagnetic fields in the millimeter band to be obtained.

The basic physics of the generation of SR pulses initiated by the radiation from the sharp edge of a current pulse can be understood from the fundamental model shown in Fig. 1(a). We will assume that an extended electron bunch with length $l_{b}$ moves with the longitudinal velocity $v_{0}=\beta_{0} c$ along a strong magnetic field in a planar waveguide with periodically corrugated walls: $b(z)=b_{1} \sin (\bar{h} z)$, where $b_{1}$ is the corrugation amplitude, $\bar{h}=2 \pi / d, d$ is the corrugation period. Under the assumption of small corrugation amplitude $b_{1}<<d$ the structure of radiated field is close to a TEM mode of the regular waveguide. However, since the electric field should be perpendicular to the metal walls of the slightly curved waveguide a small longitudinal component of the electric field appears:

$$
E_{z}=\bar{h} b_{1} \cos (\bar{h} z) E_{x}
$$

Excitation of TEM mode by an electron bunch can be described by the following equation [10] :

$$
\frac{\partial^{2} E_{x}}{\partial z^{2}}-\frac{1}{c^{2}} \frac{\partial^{2} E_{x}}{\partial t^{2}}=\frac{4 \pi}{c^{2}} \frac{\bar{h} b_{1}}{b} \cos (\bar{h} z) \frac{\partial}{\partial t} J
$$

where $b$ is the gap between the waveguide walls and $J$ is the electron current.

We will represent the electron bunch as a set of macroparticles with a small length $\Delta l$ and different initial positions over the longitudinal axis. The current of the macroparticle with the initial coordinate $z_{0}$ can be written as $J=\sigma \Delta l \delta\left(t-\left(z-z_{0}\right) / v_{0}\right)$, where $\sigma=\int_{-b / 2}^{b / 2} \rho d x, \rho$ is the charge density, $\delta(x)$ is the Dirac delta-function. According to the Eq. (2) the field excited in the waveguide by the current $J$ is given by relations :

$$
E_{x}^{ \pm}=-\frac{2 \pi k_{ \pm} b_{1} \sigma \Delta l}{b} \cos \left[\omega_{ \pm}\left(t+\frac{z_{0}}{v_{0}} \mp\right)\right]
$$

where $\omega_{ \pm}=\bar{h} v_{0} /\left(1 \mp \beta_{0}\right), k_{ \pm}=\omega_{ \pm} / c$. The upper sign in Eq.(3) corresponds to radiation in the forward direction $\left(z>z_{0}-v_{0} t\right)$, and low sign to radiation in the backward direction $\left(z<z_{0}-v_{0} t\right)$. Thus a single particle moving through a corrugated waveguide radiates a high-frequency (HF) component in the direction of the electron movement and a low-frequency (LF) component in the opposite direction. The field radiated by the whole bunch is acquired by summation of the fields emitted by the individual macroparticles. In the approximation of the unperturbed motion of the 
electrons this summation is reduced to the interference of the radiation from the front and trailing edges of the electron bunch. This emission should be interpreted as spontaneous with the total radiation power in the operating TEM mode defined by:

$$
P^{ \pm}=\frac{\pi b_{1}^{2} \sigma^{2} v_{0}^{2}}{c b} \sin ^{2}\left(\frac{\omega_{ \pm}}{2 v_{0}} l_{b}\right)
$$

Significantly higher radiation power can be achieved if the particles' self-bunching is taken into account. This process is caused by the mutual influence of the electrons and, in fact, develops from the spontaneous emission mentioned above. For further analysis it is convenient to introduce new independent variables $\xi=z-v_{0} \tau, \tau=t$ in which the longitudinal coordinate is moving with the electron unperturbed velocity. In these variables substituting Eq. (3) in Eq. (1) we derive the average longitudinal force with which the selected macroparticle acts on electrons in other macroparticles:

$$
\left.\tilde{I}_{z \longmapsto>v}\right)=-\frac{\pi e k_{ \pm} \bar{h} b_{1}^{2}|\sigma| \Delta l}{b} \cos \left(\frac{k_{ \pm}\left(\xi-\xi_{0}\right)}{\beta_{0}}\right),
$$

where upper sign corresponds to the region $\xi>\xi_{0}$, and low sign - to the region $\xi<\xi_{0}$.

Thus, in the frame of our model the electron bunch is described as the gas of macroparticles interacting via the longitudinal force (5) with a profile as shown in Fig. 1(b). It is important to note that this force has an alternating sign, that leads to the development of self-bunching of the electrons. This process can be described by the averaged equations of particle motion in the form:

$$
\begin{aligned}
& \frac{d p_{i}(\tau)}{d \tau}=\sum_{j \neq i} \tilde{I}_{L\llcorner\cdots,}, \xi_{j}\left(\tau-\tilde{i}_{\lrcorner}\right\lrcorner \\
& \frac{d \xi_{i}(\tau)}{d \tau}=\frac{p_{i}(\tau)}{m \gamma_{i}(\tau)}-v_{0},
\end{aligned}
$$

where $\tau_{j i}=\left|\xi_{i}(\tau)-\xi_{j}(\tau)\right| /\left(c \pm v_{0}\right)$ is the propagation delay of the $j$-th electron radiation at the position of the $i$-th electron. The total electromagnetic field, radiated by the whole bunch, can be found as a sum of the fields (3), emitted by a single electron.

Simulations of superradiance initiated by the radiation of an electron bunch edge were performed for the electron energy of $250 \mathrm{keV}$, the line current density of $850 \mathrm{~A} / \mathrm{cm}$, the gap between the waveguide plates of $0.2 \mathrm{~cm}$, corrugation period of $0.33 \mathrm{~cm}$ and corrugation amplitude of $0.013 \mathrm{~cm}$. Under these parameters, the operating frequency was approximately $38 \mathrm{GHz}$. The current pulse duration was considered to be equal to $1 \mathrm{~ns}$. The duration of the current pulse edges was approximately $300 \mathrm{ps}$. The self-bunching of electrons arising under the influence of the averaged force (5) is shown in Fig.2(a). In the simulations it is observed that the backward wave radiation with a low frequency has the decisive influence on the particles' motion. Correspondingly, the electrons are bunched in such a way that this component of radiation is amplified. The instantaneous 
distribution of the radiated field is shown in Fig.2(b). At the beginning (at $t=590 \mathrm{ps)} \mathrm{it} \mathrm{is}$ spontaneous and caused by the radiation of the bunch front edge. Then the particles' self-bunching development leads to the amplification of the LF component and the generation of a SR pulse emitted in the $-\mathrm{Z}$ direction (at $t=740 \mathrm{ps}$ and $t=890 \mathrm{ps}$ ). It is seen that the peak power of this LF component (Fig.2(b), at $t=890$ ps) significantly exceeds the power of the spontaneous radiation (Fig.2(b), at $t=590 \mathrm{ps),} \mathrm{as} \mathrm{given} \mathrm{by} \mathrm{Eq.} \mathrm{(4).} \mathrm{At} \mathrm{the} \mathrm{same} \mathrm{time} \mathrm{the} \mathrm{amplitude} \mathrm{of} \mathrm{the} \mathrm{high-frequency}$ component radiated in the $+z$ direction remains practically constant at the level determined by the formula (4).

More detailed simulations of Cherenkov SR initiated by radiation of the electron beam edge have been performed based on the PIC (particle-in-cell) code KARAT [11]. In these simulations the geometry of the cylindrical slow-wave structure (SWS) of the Ka-band SR generator and the electron injector shown in Fig. 3 (a) coincides with that used in experiments. The beam emission onset at the voltage leading edge which rises linearly up to $-300 \mathrm{kV}$ for $300 \mathrm{ps}$ accounted for the delay of the explosive electron emission development at the cathode [12]. In these simulations, the time of the electrons" acceleration in the "cathode-anode" gap is comparable to the rise time of the voltage pulse. As a result, kinematic effects [13] provided sharpening of the current front at the entrance of the SWS (Fig. 3 (b)) by $\sim 40$ ps as compared to the voltage leading edge. Radiation from the current sharp edge becomes a seed for the development of the SR pulse (Fig. 3 (c)). PIC simulations, as well as modeling using an average approach, show that picosecond variations in the value of the current front duration (which, in turn, is defined by the front of the voltage pulse) practically doesn't influence the peak power of the SR pulse but changes significantly the time of the SR pulse formation (see the dashed line envelopes in Figs. 3(b) and 3(c)). Correspondingly, for coherent summation of SR pulses from independent sources it is necessary to provide the stability of the current front formation within picoseconds. The possibility of such accuracy was practically demonstrated in Ref.13.

To increase the power of the SR pulse, a profiling of the tubular electron beam trajectories along the force lines of the guiding magnetic field and a tapering of the SWS corrugation amplitude $[6,7]$ were applied in the simulations, as well as in the experiment. As a result, the peak power of the SR pulse of $600 \mathrm{MW}$ was close to the power of the electron pulse [6]. The transverse structure of the radiation corresponds to the excitation of the $\mathrm{TM}_{01}$ mode of the cylindrical waveguide. $\mathrm{In}$ the experiments the radiated field was transformed to a Gaussian wave beam.

Obviously synchronization of SR pulses generated in parallel channels (Fig. 4 (a)) requires adjustment and stabilization of the pulse phase-temporal structure within limits which are much less than the wave period $T_{m} \approx 26$ ps of the carrier frequency of $38 \mathrm{GHz}$. In the experiments, to achieve acceptable accuracy of the feeding of the independent cathodes, the beam injectors were powered by 
a single high-voltage driver, the pulse of which was split into four channels using an unmatched “cross-type" power divider (50 $\Omega$ split into $4 \times 48 \Omega$ ). The driver (similar to that described in Ref. [14]) had solid-state switches, possessed a stable voltage amplitude $\delta V / V_{\max } \approx \pm 0.01$, and formed four similar electron beams. The delay and steepness of the voltage fronts in each channel (Fig. 4 (b)) could be controlled independently by gyromagnetic non-linear transmission lines with adjustable saturation of the ferrite core [14]. Thus, the independent shifts of the voltage (and current) fronts were feasible to within a picosecond accuracy that is much less than $T_{m}$. Similarly to the PIC simulations, at the input of SWS the beam front had a sharpened part where the beam current rate of rise was as high as $\sim 5 \mathrm{kA} / \mathrm{ns}$ (Fig. 4(c)).

Figure 5(a) demonstrates the microwave detector signal envelope with the measurement of the radiation from one of the four SR generators in the far field zone at the axis of the 2-D antenna array. The signals from the other SR generators had nearly the same shape and represented narrow peaks resembling the simulated envelopes (Fig. 3(c)). With the summation of four SR pulses, each synchronously radiated in the form of a linearly-polarized Gaussian wave beam, the integral signal shown in Fig. 5(b) was observed at the same point of space. This measurement was made with $12 \mathrm{~dB}$ attenuation, i.e., the microwave power in the receiver was reduced by a factor of 16 . The equality of the amplitudes of the signals presented in Figs. 5(a) and 5(b) demonstrates a quadratic rise of the radiation power flux density in the maximum of the interference pattern. The reproducibility of the integral signal from pulse to pulse in Fig. 5(b) proves the stability of the SR phases of all four generators and the coherent nature of the summation. As a result, the experimentally demonstrated summation of the four SR wave beams provided the achievement in the paraxial interference maximum of the directional pattern shown in Figs. 5(c) and 5(d) of a power flux density which is equivalent to a single Ka-band microwave generator with an output power of $\left(600 \mathrm{MW} \times 4^{2}\right) \approx 10 \mathrm{GW}$. In particular, at a distance of $1 \mathrm{~m}$ from the antennas, the maximum power flux density was as high as $10 \mathrm{MW} / \mathrm{cm}^{2}$, and the strength of the electrical field attained here was $\sim 140 \mathrm{kV} / \mathrm{cm}$.

Thus, theoretical analysis and direct PIC simulations demonstrate the possibility of initiation of SR pulse generation by spontaneous emission of the electron beam leading edge. It has also been demonstrated experimentally that by division of the driving voltage pulse across parallel channels several SR sources can be synchronized to provide a two-dimensional super-powerful array of inphase generators. Obviously, in the process of coherent summation the maximum power density will grow quadratically with the increasing number of elementary radiators. This innovatory approach opens up new opportunities in the generation of microwave pulses with extremely high power density in multi-channel sources with the controlled distribution of the high-frequency phase in each of them. 
The work was supported by IEP UB RAS Project No. 0389-2014-0005 and, in part, by RFBR Grants No. 13-08-01088 and No/ 14-08-01180.

\section{References}

[1] N.S. Ginzburg, N.Yu. Peskov, A.S. Sergeev, A.D.R. Phelps, I.V. Konoplev, G.R.M. Robb, A.W. Cross, A.V. Arzhannikov, and S.L. Sinitsky, Phys. Rev. E 60, 935 (1999)

[2] I.V. Konoplev, P. McGrane, W. He, A.W. Cross, A.D.R. Phelps, C.G. Whyte, K. Ronald, and C.W. Robertson, Phys. Rev. Lett. 96, 035002 (2006).

[3] J. Benford, H. Sze, W. Woo, R.R. Smith, and B. Harteneck, Phys. Rev. Lett. 62, 969 (1989)

[4] H. Sze, D. Price, and B. Harteneck, J. Appl. Phys. 67, 2278 (1990)

[5] V.L. Bakunin, G.G. Denisov, and Yu.V. Novozhilova, Tech. Phys. Lett., 40, 382 (2014).

[6] S.D. Korovin, A.A. Eltchaninov, V.V. Rostov, V.G. Shpak, M.I. Yalandin, N.S. Ginzburg, A.S. Sergeev, and I.V. Zotova, Phys. Rev. E 74, 016501 (2006).

[7] V.V. Rostov, M.I. Yalandin, and G.A. Mesyats, IEEE Trans. Plasma Sci. 36, 655 (2008).

[8] V.V. Rostov, A.A. Elchaninov, I.V. Romanchenko, and M.I. Yalandin, Appl. Phys. Lett. 100, $224102(2012)$.

[9] J. Jackson, Classical Electrodynamics, John Wiley\&Sons Inc. New York-London, 1962.

[10] G.V. Kisunko, Electrodynamics of hollow systems, Leningrad, 1949.

[11] V.P. Tarakanov, User's Manual for Code KARAT (Berkeley Research Associates, Inc., Springfield, VA, USA, 1992).

[12] K.A. Sharypov, M.R. Ul'masculov, V.G. Shpak, S.A. Shunailov, M.I. Yalandin, G.A. Mesyats, V.V. Rostov, and M.D. Kolomiets, Rev. Sci. Instrum. 85, 125104 (2014).

[13] M.I. Yalandin, A.G. Reutova, K.A. Sharypov, V.G. Shpak, S.A. Shunailov, M.R. UImasculov, V.V. Rostov, and G.A. Mesyats, IEEE Trans. Plasma Sci. 38, 2559 (2010).

[14] A. I. Bushlyakov, S. K. Lyubutin, A. V. Ponomarev, S. N. Rukin, B. G. Slovikovsky, S. P. Timoshenkov, and S. N. Tsyranov, IEEE Trans. Plasma Sci. 34, 1873 (2006).

[15] I.V. Romanchenko, V.V. Rostov, V.P. Gubanov, A.S. Stepchenko, A.V. Gunin, and I.K. Kurkan, Rev. Sci. Instrum. 83, 074705 (2012). 


\section{Figure captions}

Fig.1 (Color online) (a) Model used for analysis of initiation of SR pulses generation by spontaneous emission from the leading edge of electron bunch moving in periodically corrugated waveguide. (b) Longitudinal profile of average force, with which the selected macroparticle acts on electrons in other macroparticles.

Fig.2 (Color online) (a) Electrons' self-bunching and (b) radiated field longitudinal profile at different moments of time.

Fig.3 (Color online) (a) Geometry of the SR generator used in PIC simulations. (b) Beam current pulses with different front steepness at the entrance of interaction space $z=3.5 \mathrm{~cm}$. (c) Formation of SR pulses initiated by radiation of the sharp edge of the current pulses.

Fig.4 (Color online) (a) Principal scheme of four-channel Ka-band SR backward wave oscillator. (b) Accelerating voltage pulse applied to each cathode in the experiment. (c) Electron beam current recorded at the entrance of interaction space, corresponding to the cross-section $\mathrm{z}=3.5 \mathrm{~cm}$ in Fig. 3 (a).

Fig.5 (Color online) (a) SR pulse recorded by microwave detector from a single generator. (b) Three stored detector's records of the sum of in-phase SR pulses from four generators. (c) Interference pattern of the radiation recorded by gas-discharge matrix panel at a distance of $1.5 \mathrm{~m}$ from an array of four SR generators. 
(a)

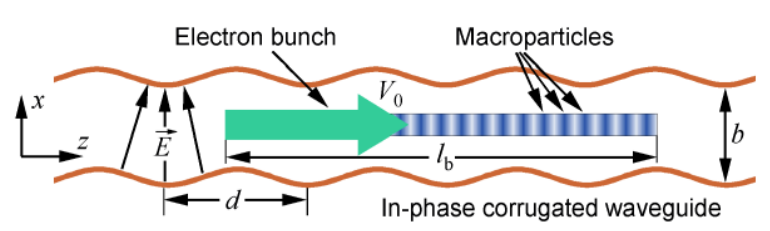

(b)

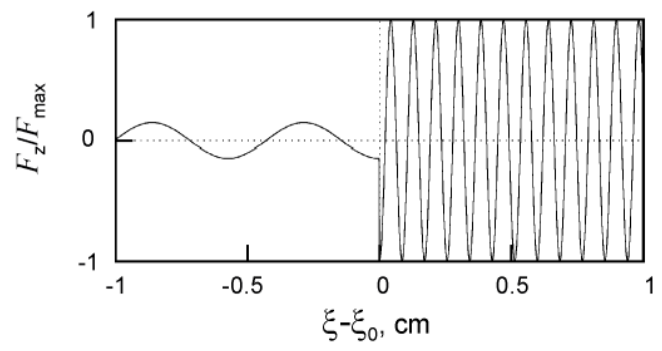

Fig. 1 

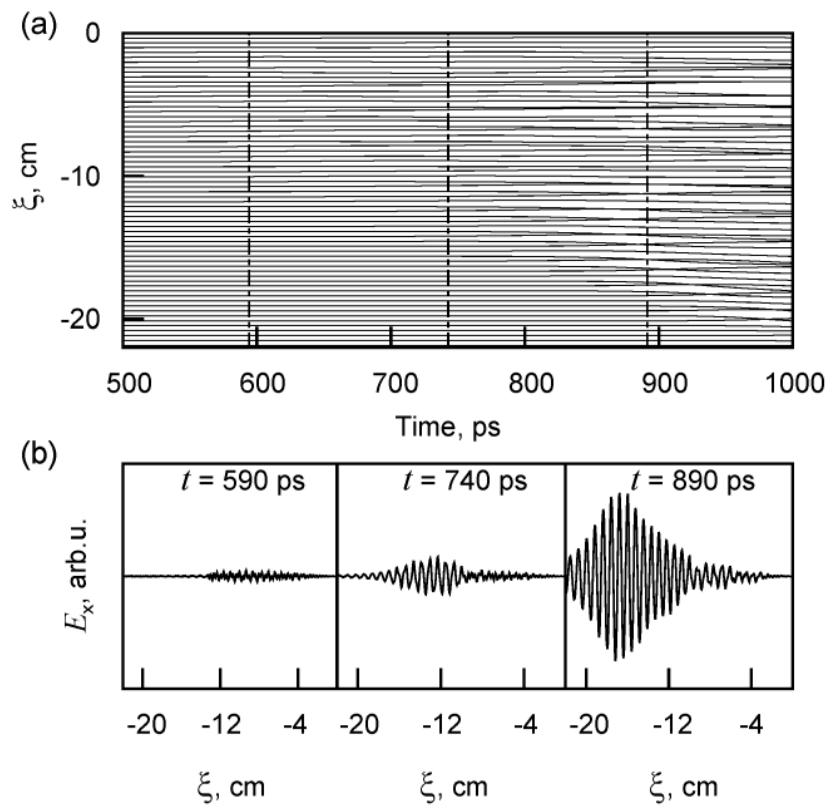

Fig. 2 
(a)

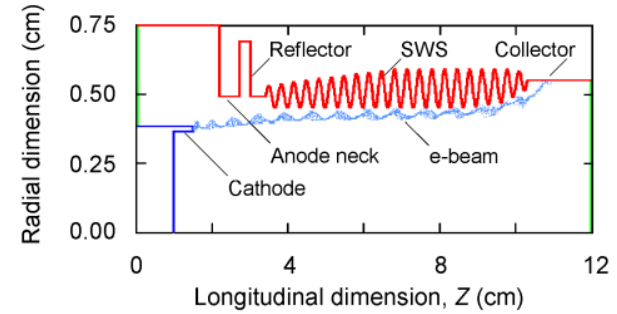

(b)

(c)
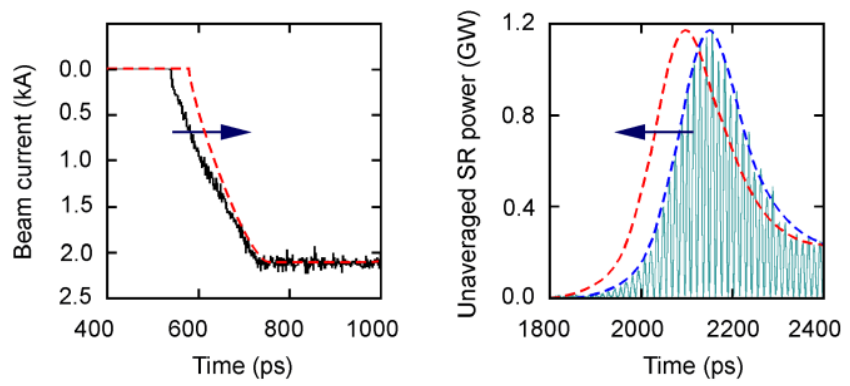

Fig. 3 


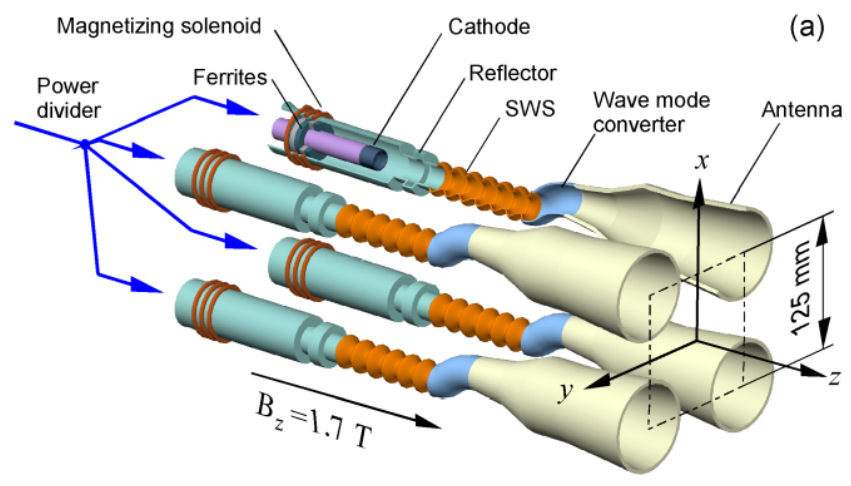

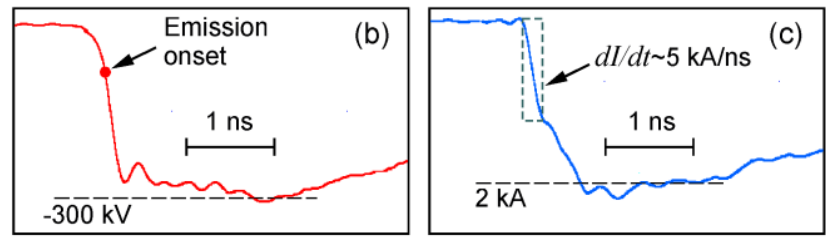

Fig. 4 


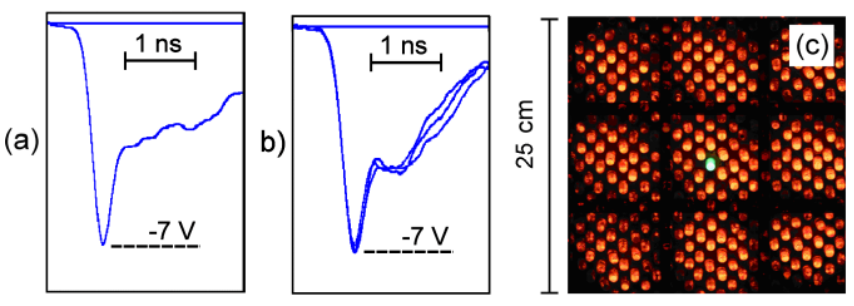

Fig. 5 